\title{
Object Searching in Scale-Space
}

\author{
Guang-Rong Ji, Bao-Liang Lu*, Xia Chen, \& Jian Wang
}

Department of Electrical Engineering, Ocean University of Qingdao,
Qingdao, 266003, P. R. China; Email: grji@mail.ouqd.edu.cn
*Lab. for Brain-Operative Device, Brain Science Institute, RIKEN
2-1 Hirosawa, Wako-Shi, 351-0198, Japan; E-mail: lu@brainway.riken.go.jp

\begin{abstract}
One of the important problems in multi-scale analysis is the detected features evolution in scale-space. By using the Laplacian of 2-D Gaussian as the kernel, it is given that the detected zero-crossing of a corner in scale-space is an ellipse whose curvature radius is determined by the analysis scale and the slope of the corner's boundary. Based on this result, we propose an object searching method for meso-scale eddy detection in the ocean remote sensing images. The method is based on the strategy of detecting possible eddies from a large region to a small region. Instead of applying the Hough transform directly to the edge map, we first search the position of the eddies by using an ellipse fitting. Then we apply local Hough transform to each of the candidate sub-regions to detect the regions which have no eddy inside. Finally, we use image segmentation and object recognition techniques to detect precise position of the eddies.
\end{abstract}

\section{INTRODUCTION}

The multi-scale analysis method has been successfully applied in image processing and computer vision. Features in scale-space can provide rich information for image analysis. The psychophysical experiments indicate that the biological (including human) vision employs multi-channel processing for low-level analysis of sensory data. Multi-scale analysis can be treated as a method of simulation to biological vision in computer vision.

A number of works in image analysis are based on the edge and corner detection. Marr and Hilldreth [1] have shown that the localization of point discontinuities can be obtained by the zero-crossings of the signal convoluted with the Laplacian of a Gaussian in different scales. Witkin [2] presented an analysis of zerocrossings of a Gaussian-smoothed signal over a range of scales in term of the standard deviations of the Gaussian kernel. Yuille and Poggio [3] proved that in any dimension the only filter that does not create generic zero-crossings as the scale increases is the Gaussian.
Mallat studied the multi-scale edges based on the wavelet transform $[4,6,7]$. The most classical way to characterize a signal from its zero-crossings is due to Logan theorem [5]. Suppose that $\forall f(x) \in L^{2}$ and its Fourier transform has a support included in one octave intervals. It is proved that if $f(x)$ does not share any zero-crossing with its Hilbert transform, then it is uniquely characterized by its zero-crossings.

One of the important problems in multi-scale analysis is the detected feature evolution in scale-space. In general, it is related to the convolution kernel function of the filter because the Gaussian function has the features of minimum support in time-frequency domain. By using the Laplacian of the Gaussian as the kernel function, it is given out that the detected zero-crossings of a corner in scale-space is an ellipse and the curvature radius of the ellipse is determined by the analysis scale and the slope of the corner's boundary.

The meso-scale eddy detection in ocean remote sensing images is an important problem in the study of ocean dynamics. Peckinpaugh and Holyer [8] have proposed a method for extracting eddy size and position from satellite imagery of the ocean. This method is based on the Hough transform. However, a big disadvantage of the Hough transform is its large storage and computation demanding. Using the Hough transform directly on the remote sensing image means that it will take a long time, and some eddies maybe neglected. Also under many circumstances, the eddy looks more similar to an ellipse than a circle in shape.

Based on the evolution analysis of the detected edges in scale-space, we propose a method for meso-scale eddy searching in the ocean remote sensing images. The method is based on the strategy of detecting eddies from a large region to a small region. Through the curve parameter fitting on the edges that are detected in a larger analysis scale, the candidate object sub-regions can be produced efficiently. Then the Hough transform is applied to each of the candidate sub-regions to delete the regions which has no eddy inside to reduce the number of the sub-regions. Finally, the techniques of 
image segmentation and object recognition are employed to the sub-regions to sign the position of the eddy in the original image. The feature parameters of an eddy such as size, the most fitted ellipse, the direction of the eddy are also computed.

This paper is organized as follows. The detection of edge-corner in scale-space based on wavelet transform is described in section 2. Section 3 presents evolution analysis in the formation of consecutive detection. Section 4 describes the proposed object searching method. Section 5 presents experimental results. Conclusions are given in Section 6.

\section{EDGE-CORNER DETECTION IN SCALE SPACE BASED ON THE WAVELET TRANSFORM}

Typically, we select the 2-D Gaussian function as the smoothing kernel

$$
\phi(x, y)=G(x, y)=\frac{1}{2 \pi} \exp \left(-\frac{x^{2}+y^{2}}{2}\right) .
$$

Applying the Laplacian operator to $\mathrm{G}()$, we have

$$
\hat{\psi}(x, y)=\frac{\partial^{2} G_{\sigma}}{\partial x^{2}}+\frac{\partial^{2} G_{\sigma}}{\partial y^{2}} .
$$

We can prove that $\psi(x, y)$ satisfies the conditions of basic wavelet, i.e.

$$
\int_{R} \psi(x, y) d x d y=0
$$

and

$$
\int_{R} \frac{\left|\hat{\psi}\left(\omega_{x}, \omega_{y}\right)\right|^{2}}{\omega_{x}^{2}+\omega_{y}^{2}} d \omega_{x} d \omega_{y}<\infty .
$$

Then we can use $\psi(x, y)$ as the wavelet function. Denoting

$$
\xi_{\sigma}(x, y)=\frac{1}{\sigma^{2}} \xi\left(\frac{x}{\sigma}, \frac{y}{\sigma}\right),
$$

we have

$$
G_{\sigma}(x, y)=\frac{1}{\sigma^{2}} G\left(\frac{x}{\sigma}, \frac{y}{\sigma}\right)=\frac{1}{2 \pi \sigma^{2}} \exp \left(-\frac{x^{2}+y^{2}}{2 \sigma^{2}}\right),
$$

and

$$
\begin{aligned}
& \psi_{\sigma}(x, y)=\frac{1}{\sigma^{2}} \psi\left(\frac{x}{\sigma}, \frac{y}{\sigma}\right)=\sigma^{2} \nabla^{2} G_{\sigma}(x, y) \\
& =\frac{-1}{2 \pi \sigma^{4}}\left(2-\frac{x^{2}+y^{2}}{\sigma^{2}}\right) \exp \left[-\frac{x^{2}+y^{2}}{2 \sigma^{2}}\right] .
\end{aligned}
$$

The wavelet transform of $f(x, y)$ respecting to scale $\sigma$ at location $(x, y)$ is defined as

$$
\begin{aligned}
W_{\sigma} f(x, y) & =f(x, y) * \psi_{\sigma}(x, y) \\
& =\sigma^{2} \nabla^{2}\left[f(x, y) * G_{\sigma}(x, y)\right]
\end{aligned}
$$

Letting

$$
\nabla^{2}\left[f(x, y) * G_{\sigma}(x, y)\right]=0,
$$

the solutions of (9) are just the zero-crossings detected in scale-space, which can be derived using the wellknown Marr-Hilldreth operator.

For the purpose of the evolution analysis of edge-corner detection in scale-space, we employ the typical edgecorner description that is formed with intersection of two straight lines as below

$$
f(x, y)=A U(y+k x) \times U(y-k x)
$$

where $k$ is a constant that determine the angle of the acute corner and $A$ is the gray level of the image. $U(\cdot)$ is the step function with

$$
U(x)= \begin{cases}1 & , x \geq 0 \\ 0 & , x<0\end{cases}
$$

Exchanging the order of derivative and convolution in $(8)$, we have

$$
\begin{aligned}
& \nabla^{2} f(x, y) * G_{\sigma}(x, y) \\
= & \frac{\partial^{2} f(x, y)}{\partial x^{2}} * G_{\sigma}(x, y)+\frac{\partial^{2} f(x, y)}{\partial y^{2}} * G_{\sigma}(x, y) \\
= & \frac{\partial}{\partial x}\left[\frac{\partial f(x, y)}{\partial x} * G_{\sigma}(x, y)\right]+\frac{\partial}{\partial y}\left[\frac{\partial f(x, y)}{\partial y} * G_{\sigma}(x, y)\right] .
\end{aligned}
$$

Denoting

$$
E(\sigma, x, y)=f(x, y) * G_{\sigma}(x, y)
$$

with $(10)$, we have

$$
f_{y}(x, y)=\delta(y+k x) U(y-k x)+\delta(y-k x) U(y+k x) \text {, }
$$

and

$$
f_{x}(x, y)=k[\delta(y+k x) U(y-k x)-\delta(y-k x) U(y+k x)] .
$$

Then we obtain

$$
\begin{aligned}
& E_{y}(\sigma, x, y)=\frac{\partial f(x, y)}{\partial y} * G_{\sigma}(x, y)=\frac{A}{2 \pi \sigma^{2}} \times \\
& \left\{\int_{-\infty}^{\infty} \exp \left\{-\frac{(x-u)^{2}}{2 \sigma^{2}}\right\}\left[\int_{k u}^{\infty} \delta(v+k u) \exp \left\{-\frac{(y-v)^{2}}{2 \sigma^{2}}\right\} d v\right] d u\right. \\
& \quad+ \\
& \left.\int_{-\infty}^{\infty} \exp \left\{-\frac{(x-u)^{2}}{2 \sigma^{2}}\right\}\left[\int_{-k u}^{\infty} \delta(v-k u) \exp \left\{-\frac{(y-v)^{2}}{2 \sigma^{2}}\right\} d v\right] d u\right\} \\
& =\frac{A}{2 \pi \sigma^{2}} \exp \left\{-\frac{\left(x^{2}+y^{2}\right)}{2 \sigma^{2}} \int_{-\infty}^{\infty} \exp \left\{-\frac{\left(1+k^{2}\right) u^{2}}{2 \sigma^{2}} \times\right.\right. \\
& \quad\left[\exp \left\{\frac{(x-k y) u}{\sigma^{2}}\right\}+\exp \left\{\frac{(x+k y) u}{\sigma^{2}}\right\}\right] d u .
\end{aligned}
$$

Taking Taylor expansion of the items in the right side of the integration and using the result

$\int_{-\infty}^{\infty} u^{j} \exp \left\{-\frac{1}{2} u^{2}\right\} d u= \begin{cases}\sqrt{2 \pi}(j-1) ! !, & \mathrm{j} \text { is even } \\ 0 \quad, \mathrm{j} \text { is odd }\end{cases}$

we have 


$$
\begin{aligned}
& E_{y}(\sigma, x, y)= \\
& \quad H\left[\exp \left\{-\frac{(y+k x)^{2}}{2\left(1+k^{2}\right) \sigma^{2}}\right\}+\exp \left\{-\frac{(y-k x)^{2}}{2\left(1+k^{2}\right) \sigma^{2}}\right\}\right]
\end{aligned}
$$

where $H=A / \sqrt{2 \pi\left(1+k^{2}\right)} \sigma$.

Following the similar way as mentioned above, we get $E_{x}(\sigma, x, y)=k H$

$$
\left[\exp \left\{-\frac{(y+k x)^{2}}{2\left(1+k^{2}\right) \sigma^{2}}\right\}-\exp \left\{-\frac{(y-k x)^{2}}{2\left(1+k^{2}\right) \sigma^{2}}\right\}\right] .
$$

From (17) and (18), we can obtain

$$
\begin{aligned}
E_{x x}(\sigma, x, y) & = \\
k^{2} H \times & {\left[-\frac{(y+k x)}{\left(1+k^{2}\right) \sigma^{2}} \exp \left\{-\frac{(y+k x)^{2}}{2\left(1+k^{2}\right) \sigma^{2}}\right\}\right.} \\
& \left.-\frac{(y-k x)}{\left(1+k^{2}\right) \sigma^{2}} \exp \left\{-\frac{(y-k x)^{2}}{2\left(1+k^{2}\right) \sigma^{2}}\right\}\right]
\end{aligned}
$$

and

$$
\begin{aligned}
& E_{y y}(\sigma, x, y)= \\
& H \times\left[-\frac{(y+k x)}{\left(1+k^{2}\right) \sigma^{2}} \exp \left\{-\frac{(y+k x)^{2}}{2\left(1+k^{2}\right) \sigma^{2}}\right\}\right. \\
& \left.-\frac{(y-k x)}{\left(1+k^{2}\right) \sigma^{2}} \exp \left\{-\frac{(y-k x)^{2}}{2\left(1+k^{2}\right) \sigma^{2}}\right\}\right]
\end{aligned}
$$

Substituting the Laplacian operation of (8) with expression of (19) and (20) yields to the zero-crossings of $f(x, y)$, which is the result of wavelet transform in scale space,

$$
\begin{aligned}
W_{\sigma} f(x, y)=H & \times\left[(y+k x) \exp \left\{-\frac{(y+k x)^{2}}{2\left(1+k^{2}\right) \sigma^{2}}\right\}\right. \\
& \left.+(y-k x) \exp \left\{-\frac{(y-k x)^{2}}{2\left(1+k^{2}\right) \sigma^{2}}\right\}\right]
\end{aligned}
$$

\section{EVOLUTION ANALYSIS OF THE DETECTION EDGES IN SCALE-SPACE}

Let $W_{\sigma} f(x, y)=0$, the zero-crossings are described with the following implicit function

$$
\begin{aligned}
& (y+k x) \exp \left\{-\frac{(y+k x)^{2}}{2\left(1+k^{2}\right) \sigma^{2}}\right\}+ \\
& (y-k x) \exp \left\{-\frac{(y-k x)^{2}}{2\left(1+k^{2}\right) \sigma^{2}}\right\}=0 .
\end{aligned}
$$

According to (22), the dependency of the geometric fealures of the detected edge-corner curve on the scale value $\sigma$ in scale-space is described. In this section, we discuss the geometric description of the zero-crossings in term of the scale parameter. The evolution of the consecutive results of edge-corner detection in scalespace is formulated.

To characterize the geometric features of the corner curve with respect to the scale value, we take the Taylor expansion of (22) at point $(0,0)$ as follows

$$
\begin{aligned}
E^{z}(\sigma, x, y) & =E^{z}\left(\sigma, x_{0}, y_{0}\right) \\
+ & {\left.\left[\left(x-x_{0}\right) \frac{\partial}{\partial x}+\left(y-y_{0}\right) \frac{\partial}{\partial y}\right] E^{z}\right|_{\substack{x=x_{0} \\
y=y_{0} \\
\text { a }}}+\left.\frac{1}{2 !}\left[\left(x-x_{0}\right) \frac{\partial}{\partial x}+\left(y-y_{0}\right) \frac{\partial}{\partial y}\right]^{2} E^{z}\right|_{\substack{x=x_{0} \\
y=y_{0}}} } \\
+ & \left.\frac{1}{3 !}\left[\left(x-x_{0}\right) \frac{\partial}{\partial x}+\left(y-y_{0}\right) \frac{\partial}{\partial y}\right]^{3} E^{z}\right|_{\substack{x=x_{0} \\
y=y_{0}}},
\end{aligned}
$$

and then we get

$$
y^{2}+3 k^{2} x^{2}=2\left(1+k^{2}\right) \sigma^{2} .
$$

This is an elliptical equation with center of $(0,0)$. It means that the zero-crossings around the intersection of two edges can be approximated to an elliptical description. Given a corner, the curvature of the detected curve can be derived from (26).

\section{EDDY SEARCHING BASED ON THE DETECTED EDGES IN SCALE-SPACE}

The Hough transform has been recognized as a technique of almost unique promise for shape and motion analysis in images containing noisy, missing, and extraneous data [9]. It has been widely used in line, circle and ellipse detection. Eddy in remote sensing images usually is not a regular circle or ellipse. And it usually exists in an unpredicted background. So the detected edges of the remote sensing images are more or less complex in which the eddy's boundary only takes a small proportion. As mentioned above, the Hough transform has the disadvantage of requiring large storage and computation, especially in detecting ellipse that is expressed by five parameters. Therefore the correctness rate will decrease rapidly as the complexity of the edges and the number of detected ellipse increases. So it seems that it is not a suitable method to apply the Hough transform directly to the whole edge map for eddy detection. This is also indicated by our experimental results.

For the purpose of the eddy detection in ocean remote sensing images, we propose a method for searching meso-scale eddy. The method can be described as follows.

- The least square ellipse fitting is used to fit every one of the continue curves in the detected edges to an ellipse and to delete the ellipses over the meso-scale range to produce the candidate sub-regions in the original image. 
- The Hough transform is applied to the candidate subregions. The candidate sub-regions in which no eddy exists are removed. As a result, only a few number of candidate sub-regions are left.

- The techniques of image segmentation and object recognition are used to extract the position of the eddy in the candidate sub-regions.

\subsection{Ellipse curve fitting}

The boundary of the eddy is usually disconnected and made up of several arcs. To search the regions which are probable to have an eddy inside quickly, each curve is thought about separately. First polygonal approximation is used to represent each digital curve. In this way, it can not only preserve the curve local properties but also make the computation of mathematical and geometrical properties much easier. Then we fit the vertices of the approximated polygon to an ellipse according to the least square error rule.

Given the points which are the vertices of the polygon on the original curve $P_{0}, P_{1}, \ldots, P_{m}$. They could be expressed as a set of the following point pair.

$$
\left\{x_{i}, y_{i}\right\} \quad i=1,2, \Lambda, m
$$

Since an ellipse can be described by the general equation for a conic

$$
a x^{2}+b x y+c y^{2}+e x+d y+1=0
$$

with the constraint $b^{2}-4 a c<0$.

We define

$$
P(x, y)=a x^{2}+b x y+c y^{2}+d x+e y+1
$$

as the distance evaluation between the point on the original curve and the fitting ellipse curve increases monotonically away from the ellipse[10].

Let

$$
\begin{aligned}
& Q(x, y)=\sum_{i=1}^{m}[P(x, y)]^{2} \\
& =\sum_{i=1}^{m}\left[a x_{i}^{2}+b x_{i} y_{i}+c y_{i}^{2}+d x_{i}+e y_{i}+1\right]^{2}
\end{aligned}
$$

being the sum of error between the original curve and the fitted ellipse. To make the least of (27), the next equation set should be satisfied,

$$
\begin{array}{ll}
\frac{\partial Q(x, y)}{\partial a}=0, & \frac{\partial Q(x, y)}{\partial b}=0, \quad \frac{\partial Q(x, y)}{\partial c}=0 \\
\frac{\partial Q(x, y)}{\partial d}=0, & \frac{\partial Q(x, y)}{\partial e}=0
\end{array}
$$

Denote

$$
A=\left[\begin{array}{ccccc}
\sum_{i} x_{i}^{4} & \sum_{i} x_{i}^{3} y_{i} & \sum_{i} x_{i}^{2} y_{i}^{2} & \sum_{i} x_{i}^{3} & \sum_{i} x_{i}^{2} y_{i} \\
\sum_{i} x_{i}^{3} y_{i} & \sum_{i} x_{i}^{2} y_{i}^{2} & \sum_{i} x_{i} y_{i}^{3} & \sum_{i} x_{i}^{2} y_{i} & \sum_{i} x_{i} y_{i}^{2} \\
\sum_{i} x_{i}^{2} y_{i}^{2} & \sum_{i} x_{i} y_{i}^{3} & \sum_{i} y_{i}^{4} & \sum_{i} x_{i} y_{i}^{2} & \sum_{i} y_{i}^{3} \\
\sum_{i} x_{i}^{3} & \sum_{i} x_{i}^{2} y_{i} & \sum_{i} x_{i} y_{i}^{2} & \sum_{i} x_{i}^{2} & \sum_{i} x_{i} y_{i} \\
\sum_{i} x_{i}^{2} y_{i} & \sum_{i} x_{i} y_{i}^{2} & \sum_{i} y_{i}^{3} & \sum_{i} x_{i} y_{i} & \sum_{i} y_{i}^{2}
\end{array}\right],
$$

$X=\left[\begin{array}{lllll}a & b & c & d & e\end{array}\right]^{T}$,

and

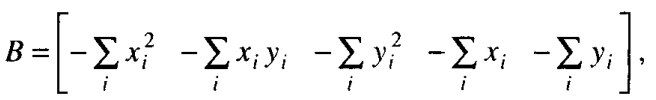

we have

$$
A X=B \text {. }
$$

From (32), we can get

$$
X=A^{-1} B \text {. }
$$

If the parameter $X=\{a, b, c, d, e\}$ in (33) satisfies the constraint $b^{2}-4 a c<0$, then it is a set of ellipse parameters. But that is still not enough. We need to compute the error between the original curve and the fitting ellipse curve. If the error is beyond a given bound, the ellipse parameter then is believed not to be the proper one. The fitting ellipses satisfying the above condition are considered as the candidate sub-regions of an eddy.

\subsection{Hough transform in the candidate sub-regions}

In the previous part, we only take information of one curve into consideration. So it is only a rough searching. As the Hough transform has an characteristic of detecting broken edges caused by noise or such circumstances that one object is covered by the other object, the Hough transform is a good mean to compute the ellipse parameter considering several edge curves simultaneously. The Hough transform is a mapping from the image plane onto the parameter space. The parameter space is quantized into an accumulator array, and each accumulator stands for the curve specified by the coordinates. For each edge point on the image plane, the curves passing through the point are computed, and the accumulators corresponding to the curves are incremented by one. After the transform, the accumulator with a peak value indicates the existence of a curve. We use the standard Hough transform for detecting ellipse in our experiment.

Because the number of edge points decrease greatly when only a local region is considered, the correctness rate and the speed of computation of detecting an eddy improve greatly. The experimental results will show that 
the region, where no eddy exists, was detected successfully. But as the boundary of an eddy is not so regular, it is probably possible to detect several ellipses in the local region in which the edge points are very complex. So it is still required a further step to extract the position of the eddy.

\subsection{Segmentation and recognition in the local region}

After the Hough transform in the local region, only a few number of candidate sub-regions are left. As a result, the image segmentation can be performed efficiently. The technique of image segmentation proposed by Ostu [11] is used to separate the eddy from its background. Two binary mathematical morphology operations, i.e., erosion and dilation, are applied to the segmented regions to delete the small-area regions, to break the connection between the regions, and to smooth the boundary of the region. All the regions are labeled for recognition. We defined three features to recognize the eddy region. The most important feature is the shape parameter $C$ that is defined as

$$
C=\frac{L^{2}}{4 \pi A^{2}}
$$

where $L$ is the perimeter of a region and $A$ is the area of a region. As an eddy is similar to a circle or an ellipse in shape, the feature parameter $C$ should change around one.

In addition to the feature $C$, two other supplementary features are defined. One is the area of the region, as the meso-scale eddy could not be too big or too small. The other is the ratio of central moment $M_{02} / M_{20}$ defined by

$$
M_{02}=\sum_{i=1}^{n} \sum_{j=1}^{n} f(i, j)(j-\bar{j})^{2}
$$

and

$$
M_{20}=\sum_{i=1}^{n} \sum_{j=1}^{n} f(i, j)(i-\bar{i})^{2}
$$

where $(\bar{i}, \bar{j})$ is the centroid of the region. If $M_{20}>M_{02}$, the region stretch is along $I$ axis, otherwise the region stretch is along $J$ axis. The $M_{02} / M_{20}$ value of an eddy changes around one.

From all the regions in the local area, we can select the region that is more similar to a circle or an ellipse through the three feature parameters defined above.

\section{EXPERIMENTAL RESULTS}

The experimental results are given in Fig. 1. Fig . 1(a) is the original remote sensing image and Fig. 1(b) is the extracted edge map. The fitted ellipses through curve fitting is shown in Fig. 1 (c) and the detected ellipses using the Hough transform in the candidate sub-regions is shown in Fig. 1(d). Fig. 1(e) shows the extracted meso-scale eddy in the original remote sensing image.

\section{CONCLUSION}

Based on the Laplacian of the 2-D Gaussian as the kernel functio and with a typical image which contains corner and linear edges, the evolution analysis of zerocrossings of corner in scale-space is given. The zerocrossing of the corner is an ellipse and whose curvature radius is determined by the analysis scale and the slope of the boundary of the corner.

We have proposed a meso-scale eddy searching method based on the strategy of detecting eddies from a large region to a small region. Firstly, a number of candidate sub-regions of an eddy are searched roughly. Then by using Hough transform in the local region, more accurate position of a probable eddy is signed out. Finally, the position of the eddy is extracted. The experimental results shows that the proposed method can detect the meso-scale eddy in ocean remote sensing images efficiently and accurately.

\section{REFERENCES}

[1] D. Marr and E. Hildreth, "Theory of edge detection", Proc. Royal Society of London, Vol. B 207, pp. 187-217, 1980.

[2] A. P. Witkin, "Scale space filtering: a new approach to multi-scale descriotion," S. Ullman and W. Richards, eds., Image Understading 1984, Norwood, N. J.: Ablex, 1984.

[3] A. L. Yuille and T. A. Poggio, "Scaling theorems for zero crossings," IEEE Trans. On PAMI, Vol. 8, N0. 1, pp. 15-25, Jan. 1986.

[4] S. Mallat, "Zero-crossings of a wavelet transform," IEEE Trans. On. Information Theory, Vol. 37, N0. 4, pp. 1019-1033, July. 1991.

[5] B. F. Logan Jr., "Information in the zero-crossings of bandpass signals," Bell Syst. Techn. J., Vol. 56, pp. 487 510, Apr. 1977.

[6] S. Mallat and Sifen Zhong, "Characterization of Singnals from Multiscale Edges," IEEE Trans. On PAMI,Vol. 14, N0. 7, pp. 710-732, July. 1992.

[7] S. Mallat and W. L. Hwang, "Singularity Detection and Processing with Wavelets", IEEE Trans. On Information Theory", Vol. 38, N0. 2, pp. 617-643, 1992.

[8] S. H. Peckinpaugh and R. J. Holyer, "Circle Detection for Extracting Eddy Size and Position from Satellite Imagery of the Ocean", IEEE Transaction on Geoscience and Remote Sensing, Vol 32, No 2, 267-273, 1994.

[9] J. Illingworth and J. Kittler, "A Survey of the Hough Transform", Computer Vision Graphics and Image Processing, Vol. 44, pp. 87-116, 1988.

[10] P. L. Rosin and A. W. West, "Nonparametric Segmentation of Curves into Various Representations", IEEE Trans. On PAMI, Vol. 17, No. 12, 1140-1153, 1995

[11] N. Ostu, "Threshold Selection Method from Gray-level histogram", IEEE Trans. On SMC, Vol. 9, 62-66, 1979. 


\section{ACKNOWLEDGEMENTS}

The authors gratefully acknowledge the support of the National Science Foundation of China, under the grant NSFC 69702010

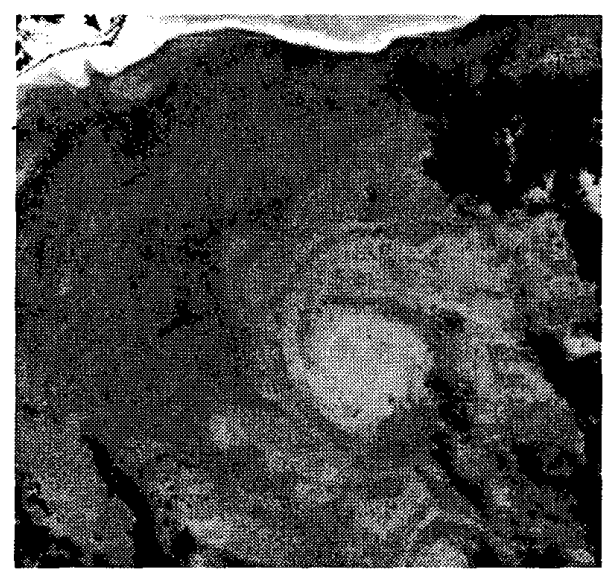

(a) Original remote sensing image

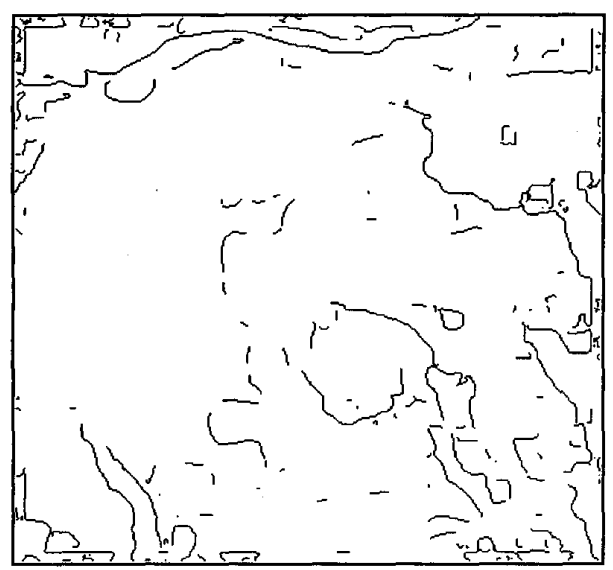

(b) The edge image

Fig 1. The experimental results

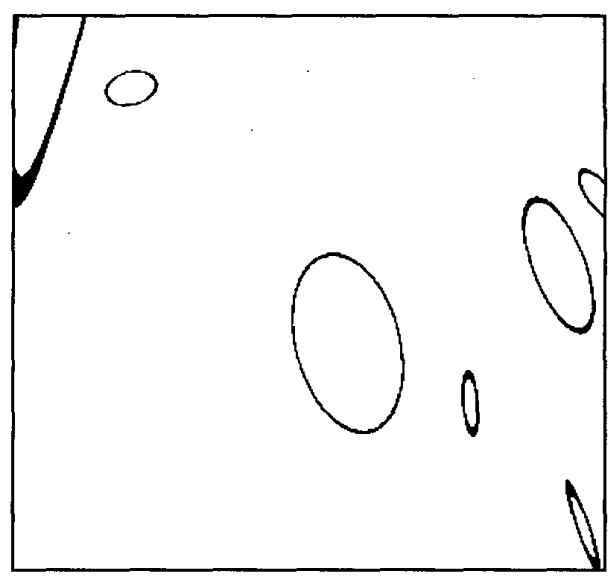

(c) The searched ellipses by using the curve fitting

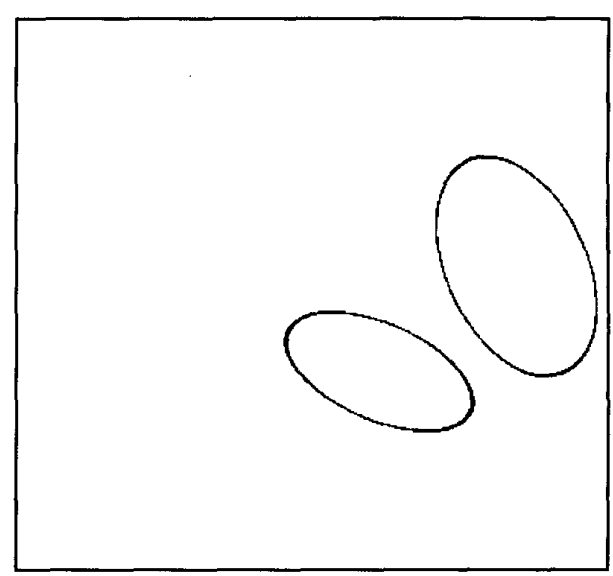

(d) The detected ellipses by using the local Hough transform

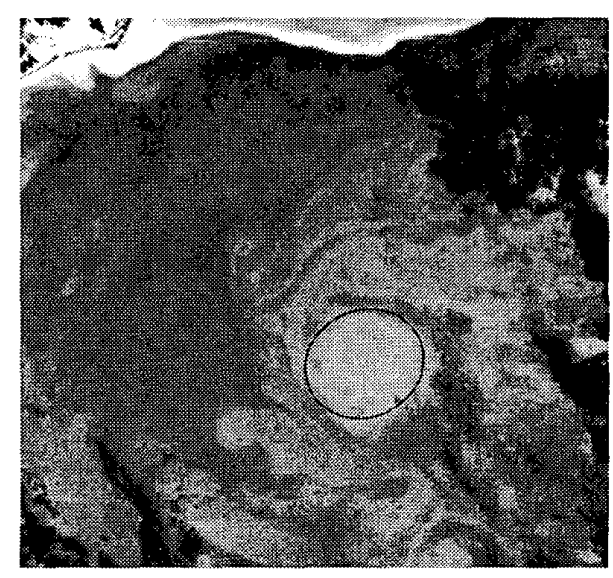

(e ) The detected eddy and its fitted ellipse 